\title{
MIDAS
}

Museus e estudos interdisciplinares

$8 \mid 2017$

Dossier temático "Objetos e museus: biografias, narrativas e vínculos identitários"

\section{Educación patrimonial para la inteligencia territorial y emocional de la ciudadanía} José María Cuenca-López e Jesús Estepa-Giménez

\section{CpenEdition}

Journals

Edição electrónica

URL: http://journals.openedition.org/midas/1173

DOI: $10.4000 /$ midas. 1173

ISSN: 2182-9543

Editora:

Alice Semedo, Paulo Simões Rodrigues, Pedro Casaleiro, Raquel Henriques da Silva, Ana Carvalho

Refêrencia eletrónica

José María Cuenca-López e Jesús Estepa-Giménez, « Educación patrimonial para la inteligencia territorial y emocional de la ciudadanía », MIDAS [Online], 8 | 2017, posto online no dia 31 julho 2017 , consultado no dia 02 maio 2019. URL : http://journals.openedition.org/midas/1173 ; DOI : 10.4000/ midas. 1173

Este documento foi criado de forma automática no dia 2 Maio 2019.

\section{(i) 8

Midas is licensed under a Creative Commons Attribution-NonCommercial-ShareAlike 3.0 International License 


\title{
Educación patrimonial para la inteligencia territorial y emocional de la ciudadanía
}

\author{
José María Cuenca-López e Jesús Estepa-Giménez
}

\section{Antecedentes del proyecto EPITEC}

1 El interés por las relaciones entre patrimonio y educación es una temática que ha venido preocupando a los miembros del equipo de investigación de este proyecto, integrados en el taller EDIPATRI, del grupo de investigación DESYM (Universidad de Huelva), desde hace aproximadamente 25 años. Sin embargo, es en 1998 cuando prácticamente el grupo empieza a configurarse y desarrollar las primeras aportaciones de forma colectiva, constituyendo el germen de lo que actualmente se ha convertido en EDIPATRI (Educación e Interpretación del Patrimonio), como referente de investigación, innovación y reflexión respecto a la educación patrimonial, con una visión interdisciplinar, un concepto holístico y simbólico-identitario del patrimonio y en el marco de un modelo didáctico de carácter constructivista y sociocrítico. Desde estas premisas se conecta la educación formal con la comunicación del patrimonio en los ámbitos no formales e informales (Cuenca-López et al. 2013).

2 En el proyecto EPITEC (2016-2019) ${ }^{1}$ pretendemos integrar estos planteamientos con los postulados de la inteligencia territorial, que se articula como una perspectiva desde la cual abordar los problemas complejos de las sociedades actuales, ya que ambiciona ser la ciencia pluridisciplinar cuyo objeto es el desarrollo sostenible de los territorios en la sociedad del conocimiento, y cuyo sujeto es la comunidad territorial. Su objetivo es impulsar, a escala de un territorio, una dinámica de desarrollo sostenible basada en una combinación de los objetivos económicos, sociales, medioambientales y culturales (Girardot et al. 2009).

3 Este enfoque promovería la inteligencia ciudadana para la toma de decisiones en un contexto de gobernanza horizontal multiescalar, para tratar las tensiones socioecológicas 
en comunidades específicas, por parte de la ciudadanía activa (Miedes, Sánchez y Moreno 2014).

4 Por su parte, la inteligencia emocional se vincula a la capacidad del individuo para tomar conciencia de sus emociones, comprender los sentimientos de los demás, fomentar la capacidad de trabajar en equipo, desarrollar una actitud empática y social y convivir en un ambiente armónico, en resumen, fomentar el desarrollo personal de carácter comunitario (Goleman 2008).

5 En este sentido el patrimonio se configura como un elemento clave para el desarrollo de estas propuestas, en las que el territorio, la ciudadanía y las comunidades sociales sean abordadas dentro del campo educativo, asociadas al ámbito de las emociones, entendidas como el tratamiento de los sentimientos y capacidades emocionales que vinculan la sociedad (y en nuestro caso al alumnado) con sus identidades, sus tradiciones, sus paisajes.

6 La conexión entre estas inteligencias, territorial y emocional, y el patrimonio es lo que configura la esencia de este proyecto, para el tratamiento y configuración de identidades territoriales y culturales a través de la educación patrimonial, desde su perspectiva simbólico-identitaria, holística, innovadora y sociocrítica, atendiendo a los problemas socialmente relevantes de la ciudadanía.

7 La educación patrimonial es una línea de investigación que está creciendo exponencialmente (Fontal y Ibáñez 2015) y entre los trabajos que relacionan patrimonio, identidad y ciudadanía, podemos resaltar las reflexiones y estudios sobre patrimonio, paisaje y ciudadanía de Brusa (2011) o los de Semedo (2015) y Pinto (2016), donde se trabaja aspectos relacionados con museos, público y ciudadanía, así como los que conectan comunicación, museo e identidad (Macdonald 2006; Davis 2007; Copeland 2009). De manera similar se pueden citar los trabajos de Martín-Cáceres y Cuenca-López (2015) o Estepa-Guiménez (2013), en los que se analizan las conexiones entre educación formal y no formal con respecto al patrimonio y la identidad y sus relaciones con propuestas de educación para la formación de ciudadanos. En la misma línea, las relaciones entre los ámbitos formales, no formales e informales en la educación patrimonial y sus conexiones con el campo de las escalas identitarias han sido objeto de estudio por Calaf (2010) o Fontal (2013), siempre trabajándose desde perspectivas interdisciplinares.

\section{Problema de investigación y objetivos generales}

8 Este estudio pretende analizar los procesos de enseñanza y aprendizaje en el ámbito de la educación formal, en conexión con las instituciones museísticas y centros de interpretación del patrimonio histórico-artístico, cultural, natural y científicotecnológico, lo que le otorga un marcado carácter interdisciplinar.

9 Por ello, el problema básico de investigación es el siguiente: ¿cuáles y cómo son las conexiones existentes entre las instituciones escolares y los centros patrimoniales para el desarrollo de propuestas educativas tendentes a promover las inteligencias territorial y emocional en el alumnado de enseñanza obligatoria? A partir de este cuestionamiento básico se establecen como objetivos generales:

1) determinar las características básicas de las propuestas didácticas que conectan desde el ámbito formal las instituciones escolares y las patrimoniales, para la formación en una ciudadanía crítica y participativa; 
2) conocer los aportes que la educación patrimonial puede hacer para trabajar las inteligencias territorial y emocional (paisajes, identidad, ciudadanía, territorio...) en la enseñanza obligatoria;

3) detectar los obstáculos existentes para que se desarrollen propuestas educativas tendentes a estas conexiones, anteriormente indicadas;

4) diseñar, experimentar y evaluar propuestas curriculares desde el paradigma didáctico actual en el campo de la educación patrimonial;

5) contrastar los resultados obtenidos de los estudios realizados entre las instituciones de los diferentes países participantes.

Siguiendo estas premisas, esta investigación consta de un amplio equipo de investigación (cuadro 1) y se articula mediante el análisis de la situación de la educación patrimonial a nivel internacional, tanto en Europa (España, Portugal e Italia) como en América (Argentina, Chile y Estados Unidos), seleccionando países que cuentan con tradición en estos estudios y que están conformados por sociedades de carácter intercultural, donde las relaciones de identidad juegan un papel crucial.

\begin{tabular}{|l|l|l|}
\hline PAIS & COORDINADORES/AS & EQUIPO DE INVESTIGACIÓN \\
\hline \multirow{5}{*}{ ESPAÑA } & $\begin{array}{l}\text { José María Cuenca-López (IP1), Jesús } \\
\text { Estepa-Guiménez (IP2), Roque } \\
\text { Jiménez, Mángeles de las Heras, } \\
\text { Gabriel Travé, Blanca Miedes, Myriam } \\
\text { J. Martín (Univ. Huelva), Roser Calaf } \\
\text { (Univ. Oviedo) }\end{array}$ & $\begin{array}{l}\text { Inmaculada Gómez, Emilio Delgado, } \\
\text { Romero, Daniel Abril, Inmaculada López, } \\
\text { Arias (Univ. Huelva), Mario Ferreras (Univ. } \\
\text { Sevilla), Laura Lucas (Univ. Valladolid) }\end{array}$ \\
\hline ITALIA & $\begin{array}{l}\text { Beatrice Borghi (Univ. Bologna), Elena } \\
\text { Musci (Univ. Basilicata) }\end{array}$ & $\begin{array}{l}\text { (Univ. Bologna), Rossella Andreassi (Univ. } \\
\text { Molisse), Marta Brunelli (Univ. Macerata), } \\
\text { Maria Laura Spano (M.useo dei Ragazzi) }\end{array}$ \\
\hline PORTUGAL & Alice Semedo (Univ. Porto) & $\begin{array}{l}\text { Rafaela Ganga, Inês Amorim, Inês Ferreira } \\
\text { (Univ. Porto) }\end{array}$ \\
\hline CHILE & Carlos Muñoz (Univ. Concepción) & $\begin{array}{l}\text { Nelson Vásquez, Gabriela Vásquez (PUC } \\
\text { Valparaíso) }\end{array}$ \\
\hline EEUU & $\begin{array}{l}\text { Daniel Schugurensky (Arizona State } \\
\text { Univ.) }\end{array}$ & \\
\hline
\end{tabular}

Cuadro 1 - Miembros del equipo de investigación del proyecto

\section{Fundamento y enfoque metodológico de la investigación}

11 La estructura que presenta la investigación se centra en el paradigma interpretativo/ naturalista, bajo el cual desde una metodología cualitativa de carácter comparativo (entre instituciones patrimoniales y educativas, tipologías patrimoniales y países) se centra, 
fundamentalmente, en dos métodos: el etnográfico (Flick 2004) y el estudio de caso (Stake 1998), utilizando múltiples técnicas para la obtención de datos, como son las entrevistas (estandarizadas y en profundidad), diario de campo, la observación (sistemática y participante), los grupos de discusión (con la participación de investigadores/as y profesionales educativos expertos) y la documentación, materiales y producciones desarrolladas en el proceso de experimentación de las propuestas de educación patrimonial.

El proyecto cuenta con cinco fases de trabajo: 1) búsqueda de información y documentación; 2) detección de buenas prácticas; 3) diseño de propuestas didácticas; 4) experimentación e intervención en el centro educativo y patrimonial; y 5) evaluación de las propuestas y establecimiento de conclusiones finales del estudio. Acualmente se está cerrando la segunda fase y se ha comenzado la tercera, para diseñar con la información obtenida hasta ahora propuestas didácticas que se experimentarán durante el año 2018.

\section{Fuentes de información}

13 Las fuentes de información para el desarrollo de esta investigación son todos los materiales producidos en el aula para el desarrollo de las experiencias de educación patrimonial que son objeto de estudio, así como las propias observaciones de las intervenciones de aula, realizadas en las fases de trabajo de campo. A ello hay que unir todos las sesiones de grupos de discusión en las que participan los miembros del equipo de investigación con los/as profesionales de la educación, expertos en el tratamiento educativo del patrimonio.

La investigación parte de las 29 instituciones patrimoniales participantes en el estudio, seleccionadas a partir de la información obtenida las fases previas del proyecto (cuadro 2). Estos centros patrimoniales, que atienden a diversas tipologías patrimoniales (histórico, artístico, etnológico, científico y natural), deben responder a unos criterios didácticos específicos, dentro de los parámetros establecidos como más adecuados para el desarrollo de propuestas de educación patrimonial: básicamente el tratamiento social del patrimonio, cercano a la visión de los museos de carácter comunitario, que responden a aspectos identitarios y de conformación de ciudadanía, o que han acreditado el diseño y realización de buenas prácticas en educación patrimonial, mediante los estudios previos llevados a cabo.

\begin{tabular}{|l|l|}
\hline INSTITUCIÓN & PAÍS \\
\cline { 1 - 2 } ASU Museum of Art (Tempe, Arizona) & $\begin{array}{l}\text { Estados } \\
\text { Unidos }\end{array}$ \\
\cline { 1 - 2 } Tempe History Museum (Arizona) & \multirow{2}{*}{ España } \\
\cline { 1 - 1 } Arizona Natural History Museum (Mesa, Arizona) & \\
\cline { 1 - 2 } Museo de Huelva &
\end{tabular}




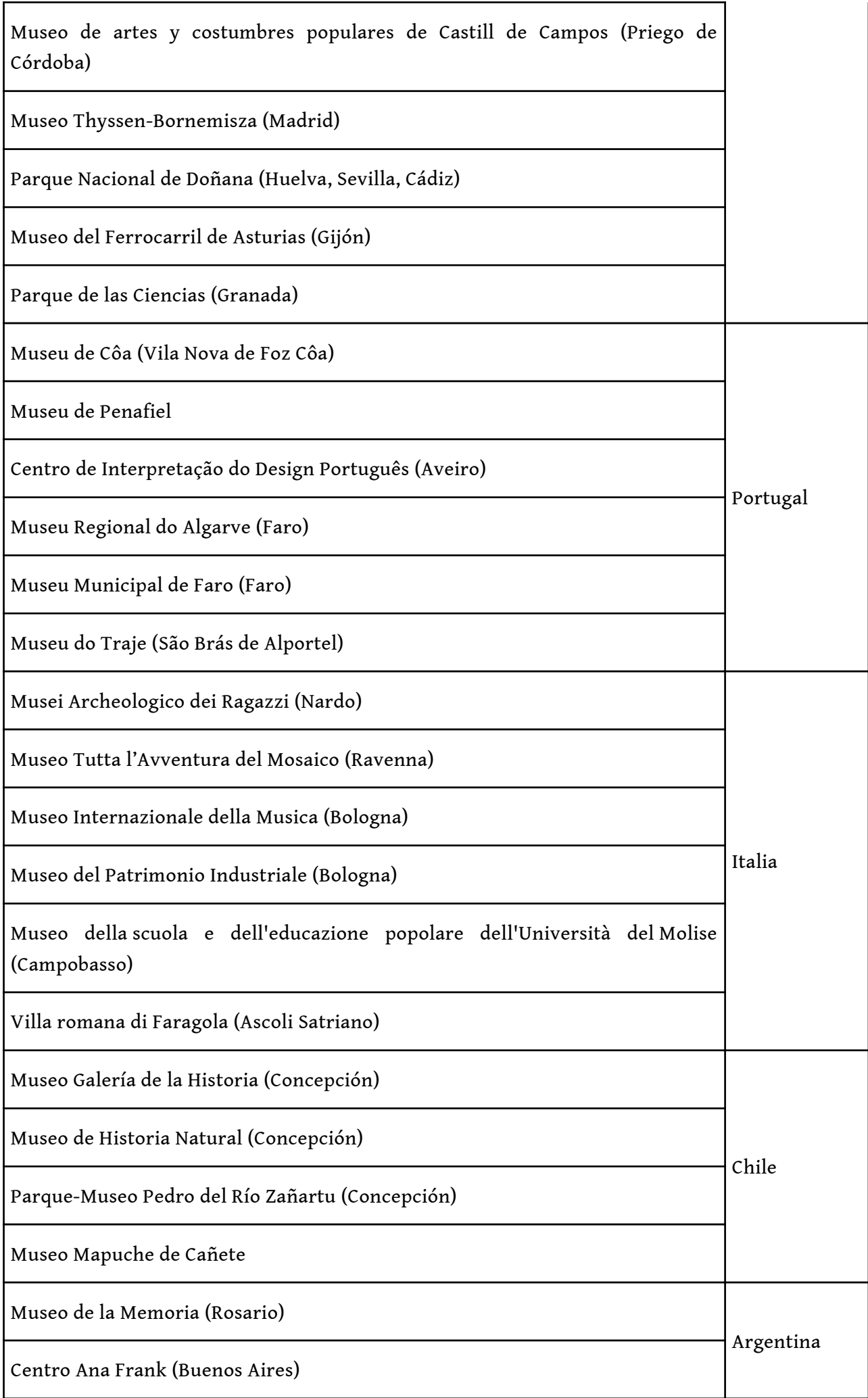

Cuadro 2 - Propuesta de centros patrimoniales participantes en el estudio 


\section{Instrumentos de análisis y toma de datos}

Para el desarrollo del proyecto se han diseñado unos instrumentos básicos de referencia, concretamente unas tablas de categorías, subcategorías, indicadores y descriptores, que se sintetizan en el cuadro 3 y unos instrumentos de registro de datos y observación que han sido ampliamente empleados con éxito en los estudios referidos. Todos ellos constituyen el fundamento metodológico del trabajo en esta investigación.

\begin{tabular}{|c|c|}
\hline CATEGORÍAS & SUBCATEGORÍAS \\
\hline \multirow{2}{*}{ I. ¿Para qué enseñar? } & $\begin{array}{l}\text { 1. ¿Hacia qué enfoques se orienta la } \\
\text { propuesta? }\end{array}$ \\
\hline & 2. ¿Qué finalidad tiene el proceso educativo? \\
\hline \multirow{5}{*}{ II. ¿Qué se enseña? } & 3. ¿Por qué algo es patrimonio? \\
\hline & 4. ¿Qué patrimonio se enseña? \\
\hline & $\begin{array}{l}\text { 5. ¿Qué nivel de interrelación existe entre el } \\
\text { patrimonio que se enseña? }\end{array}$ \\
\hline & 6. ¿Cómo se integran los contenidos? \\
\hline & $\begin{array}{l}\text { 7. ¿De qué manera se contextualizan los } \\
\text { contenidos? }\end{array}$ \\
\hline \multirow{4}{*}{ III. ¿Cómo se enseña? } & $\begin{array}{l}\text { 8. ¿Qué presencia tiene el patrimonio en el } \\
\text { programa educativo? }\end{array}$ \\
\hline & $\begin{array}{l}\text { 9. ¿Qué papel juega el profesorado y } \\
\text { alumnado? }\end{array}$ \\
\hline & 10. ¿Qué tipo de actividades se desarrollan? \\
\hline & 11. ¿Qué recursos se emplean? \\
\hline \multirow{3}{*}{$\begin{array}{l}\text { IV. ¿Qué relaciones se establecen entre } \\
\text { inteligencia emocional y patrimonio? }\end{array}$} & $\begin{array}{l}\text { 12. ¿Qué dimensiones del aprendizaje se } \\
\text { establecen? }\end{array}$ \\
\hline & $\begin{array}{l}\text { 13. ¿Qué habilidades se desarrollan a través de } \\
\text { la inteligencia emocional? }\end{array}$ \\
\hline & $\begin{array}{l}\text { 14. ¿Cuáles son los elementos constitutivos de } \\
\text { la identidad/patrimonio? }\end{array}$ \\
\hline \multirow{2}{*}{$\begin{array}{l}\text { V. ¿Qué relaciones se establecen entre } \\
\text { inteligencia territorial y patrimonio? }\end{array}$} & 15. ¿Qué visión del paisaje se trabaja? \\
\hline & 16. ¿Qué visión de la ciudadanía se trabaja? \\
\hline
\end{tabular}


17. ¿Qué visión del medioambiente se trabaja?

18. ¿Qué conexión con el entorno se aborda?

Cuadro 3 - Categorías y subcategorías del estudio

\section{Impacto esperado de los resultados}

Con los trabajos ha desarrollar en este proyecto se espera obtener un impacto científicotécnico-social en los siguientes aspectos:

- Centros educativos. Desarrollo de propuestas de innovación en la enseñanza y aprendizaje del patrimonio que aborden temas claves del currículum educativo (ciudadanía, identidad, paisajes, desarrollo sostenible, problemas socio ambientales...). Estas propuestas se basarían en un catálogo de buenas prácticas y actividades didácticas experimentadas y contrastadas a nivel internacional a lo largo de la investigación. - Centros patrimoniales. Aportaciones para el diseño de proyectos educativos de museos que conecten con el ámbito educativo formal, atendiendo a los requisitos y necesidades del currículum escolar.

- Enriquecimiento del corpus teórico y práctico de la educación patrimonial, mediante la comparación y contraste de las experimentaciones y observaciones en las diferentes instituciones de los diversos países que participan en este estudio.

- Conformación de equipos de trabajo, entre profesorado y gestores patrimoniales, para el establecimiento de las conexiones necesarias entre centros educativos y patrimoniales para el desarrollo de propuestas de educación patrimonial relevantes y significativas. - Implicaciones sociales de la educación patrimonial, a los ámbitos socioeconómicos, derivados de la activación de la inteligencia territorial en los proyectos didácticos. - Desarrollo de la inteligencia emocional, mediante el establecimiento de vínculos identitarios con los elementos patrimoniales con el fin de su disfrute, defensa, conservación, promoción y socialización.

\section{BIBLIOGRAFIA}

Brusa, Antonio. 2011. "Paisaje y Patrimonio, Entre Búsqueda, Formación y Ciudadanía.” Her\&mus: Heritage and Museography 3: 80-84.

Calaf, Roser. 2010. “Un Modelo de Investigación en Didáctica del Patrimonio.” Enseñanza de las Ciencias Sociales 9: 17-27.

Copeland, Tim. 2009. "Archaeological Heritage Education: Citizenship from the Ground Up." Treballs d'Arqueologia 15: 9-20. 
Cuenca-López, José María, et al. 2013. “Patrimonio y Educación: Quince años Investigando.” En La Educación Patrimonial en la Escuela y el Museo: Investigación y Experiencias, ed. Jesús EstepaGuiménez, 13-24. Huelva: Universidad de Huelva.

Davis, Peter. 2007. "Place Exploration: Museums, Identity, Community." En Museums and their Communities, ed. Sheila Watson, 53-75. New York: Routledge.

Estepa-Guiménez, Jesús, ed. 2013. La Educación Patrimonial en la Escuela y el Museo: Investigación y Experiencias. Huelva: Universidad de Huelva.

Flick, Uwe. 2004. Introducción a la Investigación Cualitativa. Madrid: Morata.

Fontal, Olaia, y Álex Ibáñez. 2015. “Estrategias e Instrumentos para la Educación Patrimonial en España.” Educatio Siglo XXI 33 (1): 15-32.

Fontal, Olaia. 2013. La Educación Patrimonial: Del Patrimonio a las Personas. Gijón: Trea.

Girardot, Jean-Jacques, et al. 2009. Coordination Action of the European Network of Territorial Intelligence Final Scientific Report. http://www.territorial-intelligence.eu/index.php/caenti/ deliverable08

Goleman, Daniel. 2008. Inteligencia Emocional. Barcelona: Kairós.

Macdonald, Sharon, ed. 2006. A Companion to Museum Studies. Malden: Blackwell.

Martín Cáceres, Myriam José, y José María Cuenca-López. 2015. “Educomunicación del Patrimonio." Educatio Siglo XXI 33 (1): 33-54.

Miedes, Blanca, Celia Sánchez, y Antonio Moreno. 2014. "Escenarios Alternativos para las TICS y el Conocimiento Territorial en el Marco de las Transiciones Socioecológicas: Smart Cities e Inteligencia Territorial. ¿Hacia una Sociedad del ‘Buen Conocer’? En Actas del II Congreso Internacional de Estudios del Desarrollo. Huelva: Universidad de Huelva.

Pinto, Helena. 2016. Educação Histórica e Patrimonial: Conceções de Alunos e Professores sobre o Passado em Espaços do Presente. Porto: CITCEM.

Semedo, Alice. 2015. "Representações e Identidade em Exposições de Museus." Clío. History and History Teaching 41.

Stake, Robert E. 1998. Investigación con Estudios de Casos. Madrid: Morata.

\section{NOTAS}

1. Proyecto de I+D+I "Educación Patrimonial para la Inteligencia Territorial y Emocional de la Ciudadanía. Análisis de buenas prácticas, diseño e intervención en la enseñanza obligatoria" (EDU2015-67953-P), financiado por el Ministerio de Economía y Competitividad (MINECO/FEDER), 2016-2019. 


\section{RESUMOS}

El marco teórico que sustenta el proyecto de investigación EPITEC (2016-2019) se basa en una perspectiva de la educación patrimonial de carácter interdisciplinar, simbólico-identitario, sociocrítico, constructivista y sistémico. La investigación se articula en torno a unas tablas de categorías, subcategorías, indicadores y descriptores que permiten un análisis riguroso y cualitativo de la práctica, también elementos de referencia para el diseño de la propuesta didáctica y del resto de instrumentos de investigación (entrevistas, grupos de discusión, hojas de registro de datos). Con estos instrumentos, se realizan estudios de caso en museos (históricos, artísticos, etnológicos, científicos y de la naturaleza) de los países que participan en el estudio (España, Portugal, Italia, Argentina, Chile y Estados Unidos), de manera que se puedan contrastar los resultados y establecer diferencias y afinidades en relación con las buenas prácticas, pudiendo superarse los obstáculos detectados. Los objetivos del estudio se centran en determinar las características básicas de las propuestas didácticas que conectan desde el ámbito formal las instituciones escolares y las patrimoniales; conocer los aportes que la educación patrimonial puede hacer para trabajar las inteligencias territorial y emocional; detectar los obstáculos existentes para que se desarrollen propuestas educativas tendentes a estas conexiones; y diseñar, experimentar y evaluar propuestas curriculares desde el paradigma didáctico actual en el campo de la educación patrimonial. Entre los resultados esperados de este proyecto, se aportará conocimiento sobre las conexiones interinstitucionales e internacionales que permitirán la socialización del patrimonio, su integración en las redes socioeducativas y económicas de su entorno, así como el establecimiento de vínculos emocionales.

The EPITEC research project (2016-2019) is grounded in a conceptual framework from systemic, interdisciplinary, symbolic identity, socio-critical and constructive perspective of heritage education. The research is organized around tables of categories, subcategories, indicators and descriptors that allow a rigorous and qualitative analysis of the practice. On the one hand, these instruments are a reference for the design of teaching proposal to experience and the other research instruments (interviews, focus groups, data recording sheets). On the other hand, these instruments are going to be used for case studies in museums (historical, artistic, ethnological, scientific and nature) in different countries (Spain, Portugal, Italy, Argentina, Chile and the United States) in order to compare the results and establish differences and similarities in relation to best practice. The objectives of this study are focused on determining the main characteristics of the teaching proposals which connect from the formal school environment and the heritage institutions; to know the potential of heritage education can have to work with territorial and emotional intelligence issues; identify obstacles which does not let develop educational proposals; and designing, testing and evaluating curriculum proposals from the current educational paradigm in the field of heritage education. The main expected results of this project is provide knowledge related to the connections between inter institutional and international in order to develop a teaching and learning heritage which let socialization, integration into social and educational networks provide and its economic environment as well as the establishment of emotional connections. 


\section{ÍNDICE}

Palabras claves: educación patrimonial, ciudadanía, identidad, inteligencia territorial, inteligencia emocional

Keywords: heritage education, citizenship, identity, territorial intelligence, emotional intelligence

\section{AUTORES}

\section{JOSÉ MARÍA CUENCA-LÓPEZ}

Doctor en Psicopedagogía y profesor titular en el área de Didáctica de las Ciencias Sociales en la Universidad de Huelva (Espanha). Es co-investigador responsable del proyecto de investigación EPITEC, financiado por el Ministerio de Economía y Competitividad (MINECO/FEDER) y resposable del grupo de investigación DESYM (HUM 168 del Plan Andaluz de Investigación). Es director del máster oficial interuniversitario “Investigación en la Enseñanza y el Aprendizaje de las Ciencias Experimentales, Sociales y Matemáticas” (Universidad de Huelva - Universidad Internacional de Andalucía).

Departamento de Didácticas Integradas, Facultad de Educación, Psicología y Ciencias del Deporte, Universidad de Huelva, Avenida Tres de Marzo, s/n, 21007, Huelva, España, jcuenca@uhu.es

\section{JESÚS ESTEPA-GIMÉNEZ}

Doctor en Historia y catedrático en el área de Didáctica de las Ciencias Sociales en la Universidad de Huelva (Espanha). Es co-investigador responsable del proyecto de investigación EPITEC, financiado por el Ministerio de Economía y Competitividad (MINECO/FEDER). Es director del programa de doctorado interuniversitario "Investigación en la Enseñanza y el Aprendizaje de las Ciencias (Experimentales, Sociales y Matemáticas) y de la Actividad Deportiva (IEACAD)” (Universidad de Huelva - Universidad de Extremadura).

Departamento de Didácticas Integradas, Facultad de Educación, Psicología y Ciencias del Deporte, Universidad de Huelva, Avenida Tres de Marzo, s/n, 21007, Huelva, España, jestepa@uhu.es 